Pulsar Astronomy - 2000 and Beyond

ASP Conference Series, Vol. 202, 2000

$M$. Kramer, N. Wex, and R. Wielebinski, eds.

\title{
Extreme scattering of pulsars
}

\author{
Mark A. Walker \\ Special Research Centre for Theoretical Astrophysics, \\ School of Physics, University of Sydney, NSW 2006, Australia
}

\begin{abstract}
Extreme Scattering Events are radio-wave lensing events caused by AU-sized concentrations of ionised gas. Although they were discovered more than a decade ago we still have no clear picture of the physical nature of the lenses. To discriminate between the various models, we need to amass more information on multiple imaging phenomena. Pulsars are perfect targets for such studies: they offer six- to ten-times the information content of quasar observations, and their small angular sizes make them sensitive to distant lenses. In addition, small sourcesize means that multiple imaging can be studied even when there is little change in the total source flux, because the (weak) secondary images interfere with the primary and create periodic fringes in the spectrum.
\end{abstract}

\section{Introduction}

Extreme Scattering Events (ESEs) were discovered as a result of long-term monitoring of the radio fluxes of compact radio quasars (Fiedler et al 1987). The events themselves are characterised by dramatic, frequency-dependent flux variations occurring within a period of a month or two. It is broadly agreed (Fiedler et al 1987, 1994; Romani, Blandford \& Cordes 1987; Clegg, Chernoff \& Cordes 1988) that ESEs are a due to ionised gas drifting across the line-of-sight, and that the transverse dimensions of the ionised region are only a few AU. If this scale is also representative of the longitudinal dimension of the ionised gas i.e. it takes the form of a quasi-spherical blob - then the mere existence of such regions presents us with a severe problem: the necessary electron density is $\sim 10^{3} \mathrm{~cm}^{-3}$ and at $10^{4} \mathrm{~K}$ this implies pressures $\sim 10^{3}$ times larger than is typical of the Interstellar Medium (ISM). How can such regions exist?

One possible solution to this conundrum was advanced by Walker \& Wardle (1998): a neutral gas cloud of radius $\sim$ AU would develop a photo-ionised wind, as a consequence of the ambient Galactic radiation field, with about the right electron density at the ionisation front. Furthermore, the light-curves for such a lens offer a good representation of the data for the best known example of an ESE. However, if this interpretation is correct, then it follows that the neutral gas clouds must comprise a large fraction of the mass of the Galaxy, i.e. they form a major component of the Galactic dark matter. The success of this picture as a model for ESEs provides a strong incentive for studying ESEs in detail, and with renewed vigour. This paper offers some ideas on how this can best be achieved, arguing that pulsars are by far the best targets for such studies. 


\section{Observables}

In designing experiments to study lenses, it is helpful to first set-out what quantities we might be able to observe during a lensing event. There are, in principle, six measurable quantities of interest for each image of any small-diameter source: the size, shape and orientation of the image; the location of the image; and the delay of the image. If $n$ is the refractive index, and $N \equiv \int \mathrm{d} s(1-n)$ is its lineof-sight integral, then these measurables are determined, respectively, by $\partial_{i} \partial_{j} N$ (three independent quantities; $i, j$ denote the transverse coordinates), $\partial_{i} N$ (two quantities), and a combination of $N$ and $\left(\partial_{i} N\right)^{2}$. The image size (solid angle) is perhaps the most basic measurable as it determines the received flux; the image magnification is just the ratio of apparent source sizes with/without the lens present. Because the lenses are only milli-arcseconds in size, measurement of image location, shape and orientation is only possible with VLBI, if at all. Measurement of the time delay requires a temporal variation in the intrinsic brightness of the source, and pulsars are eminently suitable.

An important point about the foregoing is that single dish (or compact interferometer) observations, which cannot hope to resolve the individual images on the sky, are enormously more informative if pulsars are targeted rather than quasars. The information gain is not simply a factor of two, but rather it is a factor of two for every image present, and it may be expected that three or five images (always an odd number) could be present during an ESE, so a factor of six or ten is possible in principle. The reason for this gain is that the presence of a delay allows the images to be resolved in time, whereas without the time dimension one only knows the total magnification (i.e. summed over all images). In practice it might not prove possible to separate all of the images, but the point remains that pulsars are much more informative than quasars when it comes to single-dish observations. In turn this means that we can distinguish between ESE models far more effectively with pulsars than with quasars.

\section{Why pulsars are so useful}

The considerations just given provide, in themselves, good reason to prefer pulsars as targets for studying ESEs. There are two other considerations which are important: pulsars are extremely compact, and they are steep-spectrum sources.

Their very small angular size has two benefits. First, the coherence patch of the radiation field is correspondingly large. This means that when multiple images are present, one of their manifestations is periodicity in the dynamic spectrum - a consequence of interference between two ray paths. This phenomenon is of great utility: a relative delay between images of order a microsecond, say, would be straightforward to observe as a $\mathrm{MHz}$ periodicity (even for glitching pulsars), but would be a tall order to detect in pulse time-of-arrival residuals (even for a millisecond pulsar; cf. Cognard et al 1993). Another big advantage of utilising pulsar dynamic spectra is that one can study very weak (low magnification) secondary images, because the power resident in periodic interference fringes is the cross-power between two images, and is proportional to the geometric mean of their magnifications. This point has been beautifully illustrated 
by Rickett, Lyne \& Gupta's (1997) study of the dynamic spectra of B0834+06, in which secondary images of magnification $\sim 0.1 \%$ were revealed.

The second benefit of small angular size is that one retains maximum sensitivity to lenses of small angular diameter; if a lens were apparently much smaller than the background source, then it would have little detectable effect. Compact radio quasars have angular sizes of order a milli-arcsecond, typically; this is comparable to the size of the lenses (at a distance of a few $\mathrm{kpc}$ ), so the Fiedler et al (1994) data set is unlikely to have provided a complete census, and could conceivably have missed a large fraction of the lens population! In the case of pulsars, the intrinsic angular diameter is so small that the observed size is always limited by interstellar scattering, i.e. diffraction caused by density inhomogeneities in the ionised ISM (e.g. Rickett 1990). This is as good as we can hope to achieve.

The fact that pulsars are steep-spectrum sources is also beneficial in that they are easily studied at long wavelengths, $\lambda$, where rays are refracted through large angles. Indeed, because $N \propto \lambda^{2}$, the cross-section for multiple imaging increases roughly as $\lambda^{4}$ at long wavelengths. Interpretation of this fact requires care, because multiple imaging events are usually recognised as such only if they split the images by more than the angular size of the image, i.e. the scattering disk in the case of pulsars. (In particular this is true of the spectral periodicities mentioned earlier.) As the scattering disk size also scales as $\lambda^{2}$, roughly, the apparent incidence of multiple imaging may be wavelength-independent. One point to bear in mind is that refraction through larger angles means that power is spread over larger physical areas in the plane of the observer, so the observed flux is smaller. Thus large lensing cross-sections go hand-in-hand with secondary images which are, typically, weak.

\section{Finding events}

One of the main barriers to studying ESEs is that they are rare events: Fiedler et al (1994) estimated that the lenses cover only $\sim 5 \times 10^{-3}$ of the (extragalactic) sky, requiring many source-years of monitoring for every event. The burden of such a monitoring program can be lightened considerably if it is acceptable to observe only infrequently, rather than the daily sampling of Fiedler et al (1994), but if one relied upon flux monitoring, then the signature of an ESE might well be missed if sampling were infrequent. Here, again, pulsar dynamic spectra prove useful: if periodicities are present then we know, without reference to any other data, that multiple imaging is occurring; this phenomenon (with weak secondary images) is expected, in essentially all lens models, as a preand post-cursor of ESEs. (ESEs can be thought of as multiple imaging events in which the primary image magnification reaches values far from unity.) The following strategy thus suggests itself. Monitor pulsar dynamic spectra for the presence of periodic fringes; the sampling interval need only be a month or so, but high spectral resolution is important ( $1 \mathrm{KHz}$, say), as the image delays could be large. If no periodicity is detected in the spectrum of a particular pulsar, at a given epoch, then that pulsar would not be expected to undergo an ESE within the next month. On the other hand, if periodicity is seen, then multiple images are present at that epoch, and there is a chance that an ESE 
will take place within the next month. It is then appropriate to activate a program of detailed multi-frequency observations, including daily acquisition of dynamic spectra, and VLBI. This strategy allows one to monitor a large sample of pulsars, achieving good sampling on the ESEs, with only a modest commitment of telescope time. For example, a total sample of 100 pulsars could be monitored monthly; a small fraction of these will display multiple imaging at any one time, so only a handful of pulsars need be observed on any given day. Not all pulsars are equally appropriate for this type of program: nearby pulsars offer little chance of the signal encountering a lens between source and observer; strongly scattered pulsars are not useful because any lens is unlikely to split the images by an angle larger than the size of the scattering disk.

\section{Signatures}

The acid test of any lens model, if not the physical model it derives from, lies in fitting the observed image properties to predictions calculated from the model $N$. However, there are also some qualitative aspects of the lensing behaviour which would help to determine the correct physical picture. In particular it is important to note that observations have not yet established the lens symmetry, if any; some symmetry does appear to be required because the light-curves are, crudely, time-symmetric. The natural choice is axisymmetry, arising from an underlying spherical symmetry; however, this immediately confronts us with the implication of an exploding lens, as discussed in $\S 1$.

Two qualitative tests for axisymmetric lenses can be given. First, if VLBI observations are able to separate the images from each other, it should be seen that all images lie on the same line (of azimuth $\phi$ ). This line should rotate systematically with time, $t$, such that $\tan \phi \propto t$, where both $\phi$ and $t$ are measured from the mid-point of the event. Secondly, for an axisymmetric lens, the geometry is effectively stationary at $t=0$, so that all image magnifications and delays should be quadratic in time around $t=0$. Even simple tests of this kind could advance our understanding of ESEs considerably.

\section{References}

Clegg A.W., Chernoff D.F. \& Cordes J.M. 1988 in Radio Wave Scattering in the Interstellar Medium, ed. J.M. Cordes, B.J. Rickett \& D.C. Backer (AIP: New York) 174

Cognard I., Bourgois G., Lestrade J.F., Biraud F., Aubry D. \& Drouhin J.P. 1993 Nat 366,320

Fiedler R.L., Dennison B., Johnston K.J. \& Hewish A. 1987 Nat 326, 675

Fiedler R.L., Johnston K.J., Waltman E.B. \& Simon R.S. 1994 ApJ 430, 581

Rickett B.J. 1990 ARAA 28, 561

Rickett B.J., Lyne A.G. \& Gupta Y. 1997 MNRAS 287, 739

Romani R., Blandford R. D. \& Cordes J. M. 1987 Nat 328, 324

Walker M. \& Wardle M. 1998 ApJL 498, L125 